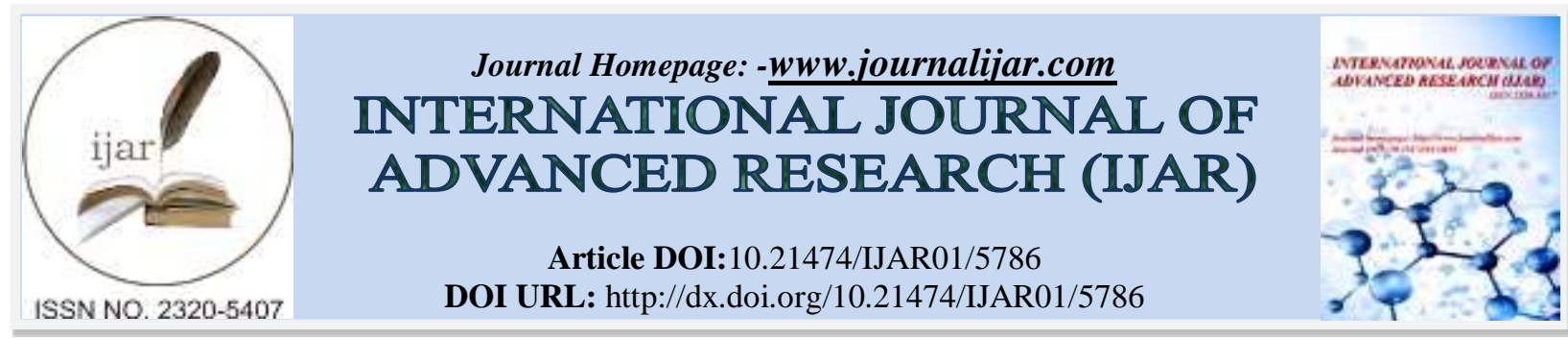

RESEARCH ARTICLE

\title{
THE ROLE OF SURGERY IN SMALL CELL LUNG CANCER.
}

\section{Bashir Ahmad Bhat ${ }^{1}$, Mushtaq Ahmad ${ }^{1}$, GhNabi Lone ${ }^{2}$ and ShiekhAejaz Aziz ${ }^{3}$.}

1. Department of surgery, Sher-I-kashmir Institute of of Medical Science (SKIMS),Srinagar, Jammu and Kashmir, India-190011.

2. Department of CVTS, Sher-I-kashmir Institute of of Medical Science (SKIMS),Srinagar, Jammu and Kashmir, India-190011.

3. Department of Medical Oncology, Sher-I-kashmir Institute of of Medical Science (SKIMS),Srinagar, Jammu and Kashmir, India-190011.

\section{Manuscript Info}

Manuscript History

Received: 06 September 2017

Final Accepted: 08 October 2017

Published: November 2017

Key words:-

Small cell carcinoma, lobectomy, tumor, survival.

\section{Abstract}

OBJECTIVE: To study the role of surgery as a primary modality of treatment for patients with small cell lung cancer (SCLC). PATIENTS AND METHODS: The study was conducted in the departments of CVTS and Medical oncology SKIMS Soura from May-2008 to Sep2010. The study was prospective /retrospective in design. All the patients $(n=30)$ who underwent pulmonary resection for LD-SCLC from Jan-2000 to Sep-2010 were included in the study. RESULTS: The median age was 56 years (range, 35-80 years). Operations performed included lobectomy in 21, bilobectomy in 3, and pneumonectomy in 6 . Postoperative therapy included chemotherapy alone in 8 patients, radiation therapy in 6 , and combined chemotherapy and radiation therapy in 10, PCI was given to 7 patients. Postsurgical tumor stage was IA ( $(n=6)$, IB $(n=6)$, IIA $(n=3)$, IIB $(n=9)$, IIIA $(n=6)$. Postoperative complications occurred in $20 \%$ of the patients (6/30), including 3 cases of supraventicular arrhythmia, 1 case of pneumonia, 1 case of prolonged air leakage and 1 case of wound site infection. Operative mortality was $3.3 \%(1 / 30)$. Patients were followed up for 1-70 months postoperatively, with a mean follow-up of 36.5 months. The 5-year survival rate according to the pathologic stage was $58.3 \%$ for patients with stage I, $25 \%$ for patients with stage II and $16.7 \%$ for patients with stage III. CONCLUSION: Pulmonary resection in patients with stage I or stage II SCLC is safe with low mortality and morbidity. Curative resection is associated with long-term survival in early stage SCLC and should be considered in selected patients.

Copy Right, IJAR, 2017,. All rights reserved.

\section{Introduction:-}

Lung cancer is most common cancer in the world, and a leading cause of death from cancer in men \& second most common in women. It accounts for nearly $13 \%$ of all new cancer diagnoses in both sexes combined. By any conventional measure, the enormity of this global problem is immense. In some countries incidence and mortality rates have peaked and are beginning to decline. In many developing nations the burden of disease is rising and will 
continue to rise because aggressive tobacco industry marketing is leading to a growing prevalence of cigarette smoking. This is also one of the major causes of cancer deaths in our valley. Lung cancer has been found to be the second commonest cancer according to a hospital-based data from Kashmir. The crude incidence rate, age standardized (world), and truncated age adjusted (40-69 years, world) incidence rates for lung cancer per 100000 population are 4.01, 6.48 and 15.28 respectively (Males 6.55, 10.09 and 23.94 respectively and females $1.19,2.14$ and 4.65$)^{1}$.

Small cell lung cancer (SCLC) accounts for approximately $15 \%$ of new cases of lung cancer diagnosed annually and for up to $25 \%$ of lung cancer deaths each year ${ }^{2}$. However, the overall incidence and mortality rates of SCLC in the United States have decreased during the past few decades ${ }^{2,3}$. Possible causes for this trend include the decrease in smoking prevalence especially in white men and a change to low-tar filters ${ }^{3}$. Recent evidence suggests that women of all ages are more likely to present with SCLC than men, and that younger women are more likely to present with SCLC than older women. Women who begin smoking at an early age are more susceptible to SCLC ${ }^{4-6}$.

\section{Material and Methods:-}

The study was conducted in the departments of CVTS and Medical oncology SKIMS Soura from May-2008 to Sep2010. The study was prospective /retrospective in design. All the patients who underwent pulmonary resection for LD-SCLC from Jan-2000 to Sep-2010 were included in the study. In all patients diagnosis was established by histologic examination of resected specimen. Some patients did not have a confirmed diagnosis of SCLC before resection and were taken to surgery for peripheral solitary pulmonary nodules suspected of being cancerous. All the patients whose pre operative diagnosis was known underwent a complete staging procedure. This included, a detailed history and physical examination, the evaluation of the performance status according to Karnofsky, a complete blood count and biochemical profile, cardiac and pulmonary function tests, chest X-ray, bronchoscopy, computed tomography of chest, the upper abdomen and brain, abdominal ultrasonography, and bone scan. Patients were classified using the TNM staging system according to the criteria established by the American Joint Committee for Cancer Staging. Operative mortality was defined as death within 30 days of operation or during same hospitalisation. The time of surgery was used as the starting date. Survival was defined as the interval between date of surgery and date of death or last follow-up. Patients who died in the perioperative period were not considered in the survival analysis. Survival for all patients was recorded upto Sep-30, 2010.

\section{Observations:-}

The study group consisted of 30 patients (27 men and 3 women). The median age was 56 (range, 35-80 years). There were 27 males and 3 female, male to female ratio was 9:1. Twenty eight patients were active smokers or had a history of smoking at the time of diagnosis; the remaining two patients were non-smokers. All the patients included in our study were symptomatic. Cough was the most common presenting symptom (30\%), followed by dyspnea $(20 \%)$, chest pain $(20 \%)$ and weight loss $(10 \%)$. All patients were classified using the TNM staging system according to the criteria established by the American Joint Committee for Cancer Staging. Most of the patients had stage I disease ; 18 patients $(60 \%)$, IA( $(n=9), \operatorname{IB}(n=9)$, stage II; 6 patients $(20 \%)$, IIA( $(n=3)$, IIB $(n=3)$, stage III; 6 patients(20\%), IIIA(n=6). Lobectomy was carried out in 21 patients $(70 \%)$, patients. Bilobectomy in 3 patients $(10 \%)$, and pneumonectomy in 6 patients (20\%). The pathologic subtype was pure small cell lung carcinoma in $21(70 \%)$ of patients, small and large cell neuroendocrine carcinoma (LCNEC) in $3(10 \%)$ of patients and small cell in combination with other tumor type in the remaining 6(20\%) cases. Post surgical pathological staging was done using the TNM staging system according to the criteria established by the American Joint Committee for Cancer Staging. Most of the patients were in stage I (40\%), stage II (40\%), and in stage III (20\%) patients. A total of 6 patients $(20 \%)$ had complications, which included supraventricular arrhythmia in 3 patients, pneumonia, prolonged air leak and wound infection in 1 each. There was only one postoperative death that of a patient with a pulmonary embolism at post operative day 13, giving a 30-day surgical mortality of $3.3 \%$. Postoperative therapy included chemotherapy in 8 patients, radiotherapy in 6, chemo-radiotherapy in 10, PCI in 7 patients and 6 patients received no adjuvant therapy including one patient who died in immediate post-op period. The follow up ranged from 6 weeks to 68 weeks, mean follow up was 36.52 months (SD 25.69). One year survival was better for female patients, it was $59.3 \%$ and $100 \%$ for males and females respectively $(\mathrm{p}=.165)$. Five year survival for females was better than that of males. For females it was $66.7 \%$ and $33.3 \%$ for males. Patients who underwent lobectomy had a 5 year survival of $42.9 \%$, bilobectomy patients had a 5 year survival of $33.3 \%$ and in pneumonectomy patients 5 year survival was $167 \%$. Patients who underwent lobectomy had a 5 year survival of $42.9 \%$, bilobectomy patients had a 5 year survival of $33.3 \%$ and in pneumonectomy patients 5 year survival was $167 \%$. Patients who received chemoradiotherapy after surgery had a 5 year survival of $40 \%$, patients receiving chemotherapy had a survival of $62.5 \%$, 
and in patients receiving no adjuvant therapy, none of the patient survived for more than 2 years. 5 year survival for post surgical stage I was $58.3 \%$, stage II was $25.0 \%$ and for stage III $16.7 \%$. A total of 6 patients had relapse, 3 patients had local relapse and 3 patients had a relapse at distant site.

\section{Discussion:-}

The most frequent localized relapse is at the site of the tumor. Even with the addition of thoracic radiation, relapse continues to occur at the primary site in up to one third of patients. ${ }^{7}$ This suggests that if survival is to improve, more aggressive local tumor control is necessary. This has led to renewed interest in the role of surgical resection of the primary tumor and regional lymph nodes.

In our study the incidence of lung cancer (SCLC) increased with increasing age. Majority of the patients (80\%) were seen in the age group 46-75 years with maximum $40 \%$ patients in age group 56-65 years. The median age was 56 years (range, 35-80 years). These observations were in accordance with the literature available. Federico Rea et al ${ }^{8}$ reported the median age of 57 years (range, 30-75 years) in their series.

In our patients overwhelming majority (90\%) were males as compared to only $10 \%$ females. The present study has revealed that mainly the males were affected with a male to female ratio of $9 / 1$ which was higher than the previous studies in western countries, but was consistent with that of the developing countries, that either lack regulations or are far less strict in regulating nicotine and tar content of cigarettes and also in which the average tar yield is higher than western countries ${ }^{3}$. This observation was contrary to most of the western literature where the incidence of SCLC among females is also on the rise due to increase in smoking ${ }^{4-6}$. Similar observations were made by HasanTatlisozet $\mathrm{al}^{9}$ the study group consisted of 141 male and 14 female patients, with a male to female ratio of $10: 1$.

In our study histopathologic profile of patients revealed $70 \%$ of patients with histopathology of pure small cell carcinoma, 10\% small cell carcinoma and large cell neuroendocrine carcinoma (LCNEC), small cell and other combinations in $20 \%$. These findings were consistent with other studies. Lim E et al ${ }^{10}$ reported that pure small cell carcinoma was present in $73 \%$ of patients whilst combined small cell carcinoma was present in $27 \%$ patients.

All operations were elective. Most of patients underwent lobectomy $(n=21,70 \%)$, because that was the preferred operation if the lesion was completely resectable, the general medical condition of the patient was acceptable, and respiratory mechanics and gas exchange were satisfactory. In 3 patients (10\%) a bilobectomy was performed and in 6 patients $(20 \%)$ a pneumonectomy was necessary due to centrally located tumors or tumors crossing the interlobar fissure affecting more than one lobe. These observations were consistent with literature. Shepherd et al ${ }^{11}$ reported 15 patients (19.4\%) had a pneuomonectomy, 46 had a lobectomy (60\%), and 2 had wedge resections. Post surgical stage was IA $(n=6)$, IB $(n=6)$, IIA $(n=3)$, IIB $(n=9)$, IIIA $(n=6)$.

In our study R0 resection was achieved in 28 patients (93.3\%) and R1 resection was achieved 2 patients (6.7\%). Similar observations were reported by A. Granetznyet al ${ }^{12}$, with R0 resection in 90 patients $(94.7 \%)$ and R1 resection in 5 patients $(5.3 \%)$.

There was one postoperative death, that of a patient with a pulmonary embolus at 13 postoperative day, giving a 30 day surgical mortality of $3.3 \%$. These findings were in accordance with other studies. Vidhan Chandra et al ${ }^{13}$ reported a operative mortality of $3 \%(2 / 77)$ in their study.

Postoperative complications occurred in $20 \%$ of the patients (6/30). There were 6 nonfatal complications, all involving the cardiorespiratory system, including 3 cases of supraventicular arrhythmia, 1 case of pneumonia, 1 case of prolonged air leakage and 1 case of wound site infection. These observations were consistent with other studies. A. Granetzny et $\mathrm{al}^{12}$ reported a postoperative complication rate of 17\% (16/95) which comprised of 6 cases of atrial fibrillation, 5 cases of atelectasis, 3 cases of pneumonia and 2 cases of wound infection.

Table 1:-showing postoperative complications

\begin{tabular}{|l|c|c|}
\hline \multicolumn{1}{|c|}{ Complications } & Frequency & Percent \\
\hline Arrhythmia & 3 & 10.0 \\
\hline Pneumonia & 1 & 3.3 \\
\hline
\end{tabular}




\begin{tabular}{|l|c|c|}
\hline Prolonged air leak & 1 & 3.3 \\
\hline Wound infection & 1 & 3.3 \\
\hline None & 24 & 80.0 \\
\hline Total & 30 & 100.0 \\
\hline
\end{tabular}

Adjuvant treatment was recorded as radiotherapy, chemotherapy, or both as appropriate. Although all patients were referred for consideration of adjuvant therapy 5 patients did not receive any additional postoperative treatment. Of the 5 patients who underwent surgery alone, 3 refused any adjuvant therapy and 2 died before completion of chemotherapy recommended by the medical oncologist. 8 patients received chemotherapy, 6 patients received radiotherapy, 10 patients received combined chemo-radiotherapy, and 7 patients received prophylactic cranial irradiation (PCI), including 2 patients with stage-I disease.

The results of our study show an overall $20.7 \%$ of relapse with only a $10.4 \%$ of relapse at the primary site alone, the remaining $10.3 \%$ of patients had a relapse at distant site alone. Federico Rea et $\mathrm{al}^{8}$ reported an overall relapse rate of $49 \%$, with a $15.4 \%$ of relapse at primary site alone. The results are similar to other studies which included surgery in multimodality therapy for SCLC.

There was only one postoperative death, with a 30-day surgical mortality of 3.3\%. The mean follow-up period of the entire study population was 36.5 months. For the 30 patients, the overall 1-, and 5-year survival rate was $63.3 \%$ and $36.7 \%$ respectively, with an estimate median survival time of 24 months. Patients were followed up for 1-70 months postoperatively, with a mean follow-up of 36.5 months.

The 5-year survival rate according to the pre-operative clinical stage was $44.4 \%$ for patients with stage I, $33.3 \%$ for patients with stage II, and $16.7 \%$ for patients with stage III $(\mathrm{p}=0.252)$. The 5 -year survival rate according to the pathologic stage was $58.3 \%$ for patients with stage I, $25 \%$ for patients with stage II and $16.7 \%$ for patients with stage III.

Regarding the impact of TNM staging on predicting the outcome in SCLC, we did not find any correlation between clinical stage and long term survival, we have to remark that $20 \%$ of patients clinically classified as stage I were understaged. On the other hand, when we consider pathologic stage, better results are obtained in stage I SCLC (T12 N0) who underwent complete surgical resection (58.3\%, 5-year survival) compared with stage II (25\% survival) and stage III (16.7\%, 5-year survival); but this did not achieve any statistical significance due to small sample size $(\mathrm{p}=0.125)$. Similar observations were made by Federico Rea et $\mathrm{al}^{8}$, who reported a 5 -year survival of $52.2 \%$ in patients with stage I disease, $30 \%$ in patients with stage II, and $15.3 \%$ in patients with stage III disease.

Table 2:-Five year survival with respect to pathologic stage

\begin{tabular}{|c|c|c|c|c|}
\hline \multirow[b]{2}{*}{ Stage } & \multicolumn{2}{|c|}{ AT 5 YEARS } & \multirow[b]{2}{*}{ Total } & \multirow[t]{2}{*}{ P value } \\
\hline & ALIVE & DEAD & & \\
\hline \multirow[t]{2}{*}{ I } & 7 & 5 & 12 & \multirow[t]{8}{*}{0.125} \\
\hline & $58.3 \%$ & $41.7 \%$ & $100.0 \%$ & \\
\hline \multirow[t]{2}{*}{ II } & 3 & 9 & 12 & \\
\hline & $25.0 \%$ & $75.0 \%$ & $100.0 \%$ & \\
\hline \multirow[t]{2}{*}{ III } & 1 & 5 & 6 & \\
\hline & $16.7 \%$ & $83.3 \%$ & $100.0 \%$ & \\
\hline \multirow[t]{2}{*}{ Total } & 11 & 9 & 30 & \\
\hline & $36.7 \%$ & $63.3 \%$ & $100.0 \%$ & \\
\hline
\end{tabular}

Another subgroup with good long-term survival was a group of 8 patients who received postoperative adjuvant chemotherapy, with overall 1-, and 5-year survivals of $82 \%$ and $62.2 \%$ respectively. Considering the subgroup of 5 patients who received no adjuvant therapy (surgery only), all died within two years of surgery. These results achieved statistical significance $(\mathrm{p}=0.001)$.

Table 3:-Five year survival with respect to Adjuvant therapy

\begin{tabular}{|c|c|c|c|c|}
\hline Adjuvant Therapy & AT 5 YEARS & \multirow{2}{*}{ P value } \\
\hline
\end{tabular}




\begin{tabular}{|c|c|c|c|}
\hline \multirow{2}{*}{ Chemo-radiotherapy } & 4 & 6 & 10 \\
\hline & $40.0 \%$ & $60.0 \%$ & $100.0 \%$ \\
\hline \multirow{2}{*}{ Chemotherapy } & 5 & 3 & 8 \\
\cline { 2 - 4 } & $62.5 \%$ & $37.5 \%$ & $100.0 \%$ \\
\hline \multirow{2}{*}{ Radiotherapy } & 0 & 6 & 6 \\
\cline { 2 - 4 } & $.0 \%$ & $100.0 \%$ & $100.0 \%$ \\
\hline \multirow{2}{*}{ NONE } & 0 & 6 & $100.0 \%$ \\
\cline { 2 - 4 } & $.0 \%$ & $100.0 \%$ & 0 \\
\hline Total & 11 & 19 & $100.0 \%$ \\
\hline
\end{tabular}

Survival was also dependent on sex. Compared with male patients $(n=27)$, female patients $(n=3)$ had a survival advantage. The 1-, and 5-year survivals for male and female patients were $59.3 \%$ and $33.3 \%$, versus $100 \%$, and $66.7 \%$ respectively $(\mathrm{p}=0.256)$. Similar observations were made by Brock et $\mathrm{al}^{14}$. The $1-, 3$-, and 5 -year survivals for male and female patients were $73 \%, 35 \%$, and $28 \%$, versus $86 \%, 63 \%$, and $60 \%$, respectively.

\section{Conclusion:-}

As long as a complete surgical resection can be performed, surgery must be considered as the first step of the multimodality treatment in these patients. Pulmonary resection in patients with stage I or stage II SCLC is safe with low mortality and morbidity. It is justified to offer primary surgery followed by chemoradiotherapy in stage T1, N0 and possibly in stage T2, N0. Prophylactic cranial irradiation should be part of the treatment program only for those patients obtaining a complete remission. Curative resection is associated with long-term survival in early stage SCLC and should be considered in selected patients.

\section{Compliance with ethical standards:-}

1. The authors declare that there is no conflict of interest.

2. A written informed consent was taken from every patient included in study.

3. All procedures performed in studies involving human participants were in accordance with the ethical standards of the institutional and/or national research committee and with the 1964 Helsinki declaration and its later amendments or comparable ethical standards.

4. The authors declare that they received no grant for the study.

\section{Bibliography:-}

1. Parvaiz A. Koul, Satish Kumar Koul, Mohammed Mushtaq Sheikh, Reyaz A. Tasleem and Azra Shah; Lung Cancer in the Kashmir valley : Lung India, vol 27, issue 3, jul-sep 2010

2. Navada S, Lai P, Schwartz AG, Kalemkerian GP. Temporal trends in small cell lung cancer: analysis of the national Surveillance, Epidemiology,and End-Results (SEER) database [abstract 7082]. J ClinOncol. 2006;24(18S)(suppl):384S.

3. Govindan R, Page N, Morgensztern D, et al. Changing epidemiology ofsmall-cell lung cancer in the United States over the last 30 years: analysis of thesurveillance, epidemiologic, and end results database. J ClinOncol. 2006;24(28):4539-4544.

4. Thompson S, Pearson MG. Changing patterns of lung cancer histology with age and gender. Thorax. 1998;53(suppl 4):A10.

5. Kmietowicz Z. Women at double risk of small cell lung cancer. BMJ.1998;317(12):1614.

6. Cartman ML, Muers MF. Rising incidence of SCLC in young women [letter]. Thorax 1999;54(7):656.

7. Perez CA, Einhorn L, Oldham RK, et al. Randomized trial of radiotherapy to the thorax in limited small-cell carcinoma of the lung treated with multiagent chemotherapy and elective brain irradiation: a preliminary report. J ClinOncol. 1984; 2:1200-1208.

8. Federico Rea, Donatella Callegaroa, Adolfo Favarettob, Monica Loya, Adriano Paccagnellab, Umberto Fantonic, GiulianaFestic, Francesco Sartoria ; Long term results of surgery and chemotherapy in small cell lung cancer: European Journal of Cardio-thoracic Surgery 14 (1998) 398-402.

9. HasanTatlýsöz, MD; M. LeventErkan, MD; SerhatFýndýk, MD; BedriKandemir, MD: Clinical Features and Outcomes of Small-Cell Lung Cancer Cases from Northern Turkey; Turkish Respiratory Journal, 2000; 2:2529. 
10. Eric Lim, FRCS (C-Th), Elizabeth Belcher, FRCS, Yoon Khoong Yap, MRCS, Andrew G. Nicholson, FRCPath, and Peter Goldstraw, FRCS ; The Role of Surgery in the Treatment of Limited Disease Small Cell Lung Cancer Time to Reevaluate : J ThoracOncol. 2008; 3: 1267-1271

11. F A Shepherd, W K Evans, R Feld, V Young, G A Patterson, R Ginsberg and E Johansen : Adjuvant chemotherapy following surgical resection for small-cell carcinoma of the lung ; Journal of clinical oncology, Vol 6, 832-838 (1988).

12. Andreas Granetzny, Ahmad Boseila,, Wolfgang Wagner, Georg Krukemeyer, Ulf Vogt c, Erich Hecker, Olaf M. Koch, FolkerKlinke ; Surgery in the tri-modality treatment of small cell lung cancer.Stage-dependent survival : European Journal of Cardio-thoracic Surgery 30 (2006) 212-216

13. VIDHAN CHANDRA, MD; MARK S. ALLEN, MD; FRANCIS C. NICHOLS III, MD; CLAUDE DESCHAMPS, MD; STEPHEN D. CASSIVI, MD; AND PETER C. PAIROLERO, MD : The Role of Pulmonary Resection in Small Cell Lung Cancer ; Mayo Clin Proc. 2006; 81(5):619-624

14. Malcolm V. Brock, MD Craig M. Hooker, MPH James E. Syphard, BS William Westra, MD Li Xu, MS Anthony J. Alberg, PhD David Mason, MD Stephen B. Baylin, MD James G. Herman, MD Rex C. Yung, MD Julie Brahmer, MD Charles M. Rudin, MD, PhD David S. Ettinger, MD Stephen C. Yang, MD ; Surgical resection of limited disease small cell lung cancer in the new era of platinum Chemotherapy: Its time has come: The Journal of Thoracic and Cardiovascular Surgery, Volume 129, jan-2005. 